https://www.journal-imab-bg.org

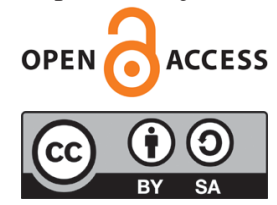

Case report

\title{
DYNAMICS OF PSYCHIATRIC SYMPTOMS IN CUSHING'S SYNDROME
}

\author{
Tatyana Telbizova ${ }^{1}$, Ivan Aleksandrov ${ }^{2}$, Mariana Arnaoudova ${ }^{1}$ \\ 1) Department of psychiatry and medical psychology, Medical University - Varna, \\ Bulgaria, \\ 2) Department of Health Care, Sliven Affiliate, Medical University - Varna, \\ Bulgaria.
}

\begin{abstract}
:
Cushing's syndrome may present with various psychiatric disorders which can lead to a misdiagnosis at the beginning, deterioration of the course of the disease and life-threatening complications. The most common psychiatric disorder is atypical depression. During the initial stages of the disease, the somatic symptoms of Cushing's syndrome can be subtle, difficult to identify, especially when the psychiatric symptoms are dominant.

Objective: The aim of the present article is to report a case of a 24-year-old woman with a Cushing's syndrome, caused by the adrenal adenoma. The onset of the disease is a suicidal attempt. Family history is significant for psychiatry disorder. The belated diagnosis led to somatic and psychiatry complications of the disease - sepsis and persisting high suicidal risk. The dynamic change in the patient's mental state - from a severe depressive episode with atypical symptoms to psychosis with single catatonic symptoms correlated well with the gradual increase of the plasma cortisol levels. The antidepressant and subsequent antipsychotic medication turned out to be ineffective. The surgical treatment, followed by a normalization of the cortisol levels was a key to the successful outcome and the reduction of self-aggression risk.

Conclusion: The early diagnosis and timely treatment of Cushing's syndrome decrease the risk of multiple complications. The shift from atypical depression to psychosis with catatonic symptoms correlates with the worsening of the hormonal imbalance. The signs of atypical depression in Cushing's syndrome, resistant to the antidepressant medication, could be considered as relatively specific symptoms, guiding specialists to its somatogenic origin for the proper diagnosis.
\end{abstract}

Keywords: Cushing's syndrome, hypercortisolemia, atypical depression, somatogenic psychosis, catatonic symptoms

\section{INTRODUCTION:}

Various psychiatric symptoms often accompany the clinical manifestation of a number of neuroendocrine disorders [1]. They refer to the exogenic, psychotic and nonpsychotic disorders. Considering their etiological relation to the somatic diseases, they are often entitled as soma- togenic or somatically determined psychoses - a term, given by K. Schneider [2].

At the beginning of the XX century, the treatment of the patients with endocrine disorders was a responsibility of general practitioners, psychiatrists, neurologists and surgeons. Although the etiology of these diseases due to hormonal imbalance had not yet been elucidated at the time, the association with various psychopathological symptoms was discovered [3]. In 1912 the neurologist and surgeon Harvey Williams Cushing (1869-1939) described a "polyglandular syndrome" that was considered the first description of the Cushing's syndrome. Twenty years later, he described not only the typical somatic symptoms of the disease but also the presence of anxiety and depressive symptoms among patients with basophilic adenoma in the adenohypophysis.

In 1940 the Swiss psychiatrist Manfred Bleuler (1903-1994) began an in-depth analysis of mood disorders originating from endocrine illnesses. Later on, in 1979, he defined the endocrinological psychiatry as a new branch of the science of mental disorders in endocrine diseases and endocrine changes in mental disorders. M. Bleuler, along with W. Stoll, focused on the undoubted overlap between the endocrine psychosyndrome and the local cerebral psychosyndrome, associated with changes in impulse, mood and inclination. Thus, the scientists proved that the endocrine diseases were not related to specific psychopathology [4]. Nowadays, the diagnosis and treatment of endocrine diseases are made much earlier. As a result, the accompanying psychopathological symptoms do not reach a level of severity that necessitates initial hospitalization in a psychiatric clinic [1, 3].

Cushing's syndrome is a rare endocrine disorder that includes a number of typical somatic symptoms, induced by increased plasma levels of glucocorticoids (hypercortisolemia). Hypercortisolemia can be of exogenic (iatrogenic) or endogenic origin. Long-term treatment with synthetic corticosteroids is the cause of exogenous Cushing's syndrome. The causes of the endogenic Cushing's syndrome are rare and they are a result of chronic overproduction of cortisol by the adrenal gland, leading to impaired feedback of the hypothalamic-pituitary-adrenal (HPA) axis and the circadian synthesis of cortisol [5]. Cushing's disease, which is due to overproduction of adrenocorticotropic hormone 
(ACTH) by a pituitary microadenoma represents around 70$80 \%$ of the cases - ACTH - dependent hypercortisolism [6]. $\mathrm{ACTH}$ - independent hypercortisolism is rare and is mainly due to adrenal gland adenoma [7]. In cases, in which the clinical manifestation of symptoms and abnormal hormonal levels of cortisol, such as in Cushing's syndrome, are not due to hypothalamic-pituitary-adrenal axis dysfunction, they are referred to as Pseudo-Cushing syndrome. In such cases, the hypercortisolemia is secondary and is caused by other factors. This is observed in a range of psychiatric disorders (depression, anxiety disorders, alcohol dependency, anorexia nervosa and bulimia) and in some somatic diseases (polycystic ovary syndrome, obesity, poorly controlled diabetes, multiple sclerosis and obstructive sleep apnea) $[8,9]$.

The somatic symptoms of Cushing's syndrome are: moon face, central obesity, proximal myopathy, thin skin with purple stretch marks and acne, hirsutism, hair loss, impaired glucose tolerance or diabetes, arterial hypertension, accelerated atherosclerosis and thromboembolism, osteoporosis, menstrual dysfunctions (incl. amenorrhea), fatigue, frequent infections (incl. sepsis). Some of these symptoms are also typical for other somatic conditions such as metabolic syndrome, polycystic ovary syndrome and insulin resistance [7].

Psychiatric disorders are also part of the clinical manifestation of Cushing's syndrome. They can be presented as mood disorders, psychotic disorders and cognitive impairment [10] and can be observed both during the active period of the disease [11] and during its remission [12]. The time from the onset of the disease to its diagnosing is an important prognostic factor for the clinical manifestation and the severity of the mental and neurocognitive dysfunctions [11]. Affective disorders are among the most commonly reported ones [13]. In milder cases, the somatic symptoms can be difficult to identify, especially when the accompanying psychiatric disorders dominate the clinical picture. The earlier the disease is diagnosed, the smaller the risk of complications and an unfavorable outcome. [7].

Cushing's syndrome is one of the most "mysterious" diseases in clinical medicine. The described somatic symptoms, interconnected in a general syndrome, are not sufficient to make the diagnosis. Appropriate hormonal evaluation is of key importance to the exact diagnosis and differential diagnosis of the disease. Imaging modalities (computer tomography (CT) scan and/or magnetic resonance imaging (MRI) of the brain and the abdomen) in search of the cortisol-producing tumors are the diagnostic methods important for the surgical treatment $[14,15]$.

The chronic overproduction of glucocorticoids can cause structural and functional changes in the brain [16]. Hypercortisolemia is the cause of atrophy of the prefrontal cortex and the hippocampus as well as the suppression of the neurogenesis in gyrus dentatus. It plays a crucial role in the pathophysiology of neurocognitive dysfunctions and depression in Cushing's syndrome, observed during the prodromal, the active periods and the remission periods of the disease [16,17].

Depression is one of the most common mental disor- ders in Cushing's syndrome. Different studies have shown that clinically manifested symptoms of depression are found in $54 \%$ [17] to $68 \%$ [18] in the studied patients. $27 \%$ of them occurred in the prodromal period of the disease [17]. Potential risk factors include female sex, advanced age, high urinary cortisol levels before treatment, and undetectable adenoma. In addition, patients with depression in Cushing's syndrome have a more severe course of the disease than those without depression. [17]. In most patients, depression has atypical clinical symptoms [11]. Atypical depression is the most common psychiatric disorder, even after normalization of the plasma cortisol levels. Atypical symptoms include increased sensitivity and irritability, affective blasts, drowsiness, fatigue, increased appetite (hyperphagia) and hypersomnia $[19,20]$. Patients with atypical depression are often comorbid with other psychiatric disorders [11]. Another feature of patients with Cushing's syndrome is their tendency to dissimulate their mental symptoms, which conceals the risk of committing suicide attempts [13].

A study found that a significant number of patients with Cushing's syndrome also reports complaints of generalized $(79 \%)$ and paroxysmal anxiety $(53 \%)$. The combination of a clinically manifested depressive episode with generalized anxiety or a panic disorder is up to $63 \%$ [18].

In addition to depressive episodes, manic and hypomanic have also been described. R. Haskett found that they occur in about $30 \%$ of patients with an affective disorder in Cushing's syndrome [13]. The cyclic Cushing's syndrome is characterized by the recurrence of episodes of overproduction of cortisol, followed by episodes with normal serum levels. During peak plasma levels of cortisol, manialike conditions have been described, mimicking bipolar affective disorder [21].

Psychotic episodes (toxic psychoses), associated with hallucinatory-paranoid experiences with or without affective symptoms and aggressive behavioral manifestations are less frequent $[13,22,23]$. Few are the described cases of Cushing's syndrome with schizophrenic syndromes, predominantly in patients with an adrenal carcinoma [23,24]. Catatonic symptoms such as hypokinesia to akinesia, mutism, negativism, mannerism, catalepsy and echolalia have also been rarely reported in patients with severe hypercortisolism and ACTH-producing neuroendocrine tumors [25].

\section{CASE REPORT:}

A 24-year-old woman was admitted in the Psychiatry Clinic in June 2019 with disorganized behavior, impulsivity and self-aggression. The patient had a degree in economics and worked as a manager. She had normal physical and psychical development during her childhood although her mother was diagnosed with placenta praevia while bearing her. That was an indication for her to be born with a Caesarean section. She had a family history of a psychiatry disorder - her mother was diagnosed with schizophrenia at the beginning of 2018 .

At the end of 2018, the patient had complaints of a suppressed mood, intense tension, expressed fatigue, difficulties in concentrating at work and pessimistic thoughts 
about her future ("it doesn't make sense to give birth to children if their fate would be just as severe"). At the same period, two of her friends had been diagnosed with severe chronic diseases, and her relationship with her boyfriend became unstable.

In February 2019, she made a suicide attempt by jumping from the fourth floor - an action planned in advance. The patient was hospitalized in an Intensive care clinic, later in Orthopedic and Traumatology department with multiple injuries. This was followed by a period of home rehabilitation and medication with Venlafaxine up to 225 mg/day, Pregabalin $225 \mathrm{mg} /$ day and Quetiapine $75 \mathrm{mg}$ /day, under the supervision of her parents. Somatic symptoms such as tachycardia (medicated with Bisoprolol $5 \mathrm{mg}$ daily), amenorrhea and hirsutism have been reported, as well as hypersomnia, increased appetite and irritability. Regardless of the treatment, anxiety was hardly affected, and the depressive symptoms persisted. Almost every day, she shared suicidal thoughts and her guilt about letting herself stay alive. She isolated herself from others and broke up with her boyfriend.

At the beginning of May 2019, there was a slight improvement in her condition - she began to communicate with friends, but the suicidal thoughts persisted. Hospitalization in an Intensive Care clinic was required due to impaired general condition from sepsis and abscesses of the right kidney, diagnosed by contrast CT (computed tomography) scan. The treatment continued in the Nephrology clinic, where hypertension and tachycardia have been diagnosed. The patient began to fall over when walking due to weakness of the proximal muscles of the limbs; the face was typically altered to "facies lunata".

The patient was consulted with an endocrinologist The CT scan was reviewed once again and a left adrenal adenoma with a size of $25 / 28 \mathrm{~mm}$ was identified. Hormonal examination showed cortisol levels $968,4 \mathrm{nmol} / \mathrm{l}$ tested in 10 P.M.; cortisol rhythm - 978,41 nmol/1 (tested 8 A.M.), 993,58 (10 P.M.), free cortisol in the urine $>2999,0 \mathrm{mkg} / 24$ h (55,5 - 286); ACTH $<5,0 \mathrm{pg} / \mathrm{mL}$ (up to 46). The treatment continued in the Endocrinology clinic, where her behavior became disorganized and unpredictable. The patient was psychomotorically tense, impulsive, irate, and negative regarding treatment, declaring that she wanted to die, with sudden episodes of self-aggression and aggression towards her parents.

The patient was admitted urgently to a psychiatric department where auto-aggression in the morning hours, accompanied by an active negativism, hostility and dysphoric abreactions were recorded. In addition to the gross disorganization of behavior and individual catatonic symptoms (mask-like facial expression, facies oleosa, active negativism, sudden agitation), the paralogical thoughts, the depressive mood and the persistent intensive suicidal plans and intentions were also noteworthy. Regardless of the psychotropic medication - Haloperidol $15 \mathrm{mg} /$ day i.m., Diazepam $20 \mathrm{mg}$ /day and Sodium valproate $2000 \mathrm{mg}$ /day p.o., no significant change in the patient's psychotic state was observed. Sedation was finally achieved during the last two days of her stay, after the administration of Promåthazine hydrochloride $75 \mathrm{mg} /$ day i.m. Yet, the impulsivity, the cognitive dysfunctions, the catatonic symptoms and the suicidal ideations were not ameliorated.

By the end of the ten-day inpatient stay, the risk of aggression and auto-aggression remained high. After subsequent laparoscopic adrenalectomy of the left adrenal gland, the patient became psychomotorically stable, with persisting depressive symptoms. The cortisol plasma levels decreased to $60,70 \mathrm{nmol} / \mathrm{l}$, cortisol urine levels $-61,98 \mathrm{mkg} /$ 24 h. Medication therapy continued with Hydrocortisone, Aripiprazole $15 \mathrm{mg} /$ day and after two weeks - Duloxetine $90 \mathrm{mg} / \mathrm{morning}$, Aripiprazole $5 \mathrm{mg} / \mathrm{evening}$ and Pregabalin $225 \mathrm{mg} /$ day.

By the end of August 2019, the levels of plasma cortisol and ACTH had normalized and the Hydrocortisone treatment was discontinued.

The patient's mood improved, she became socially active, started sports activities. Her suicidal ideations gradually disappeared as well as the somatic symptoms (facies lunata, hirsutism, amenorrhea, proximal muscle weakness etc.).

In October 2019, the Pregabalin treatment was discontinued. Being on a supportive treatment of Duloxetine $90 \mathrm{mg} / \mathrm{morning}$ and Aripiprazole $5 \mathrm{mg} /$ evening, she went back to work and successfully continued her master's degree in economics. At present, the patient's condition is monitored regularly by an outpatient psychiatrist.

\section{DISCUSSION:}

In the presented case, the first clearly manifested symptoms of Cushing's syndrome are psychiatric - severe depressive episode continuing with a suicide attempt.

Initial somatic symptoms of amenorrhea and hirsutism, which are more common in polycystic ovarian syndrome, delay the diagnosis of Cushing's syndrome. The timely detection of the hypercortisolemia would direct to the right diagnosis of Cushing's syndrome and to prevent consequent life-threatening complications [26]. The family history of schizophrenia, the pathological course of her intrauterine development and the experienced psychotraumatic events focus on an endogenic origin of the depression. Moreover, the presence of atypical symptoms of depression and the severe suicide attempt, further point towards bipolar depression than to a unipolar one [19]. Initially, the psychiatric symptoms of the severe depressive episode were more pronounced than somatic ones. Whereas life experiences of stress are risk factors not only for the manifestation of affective disorders, some authors find a relationship between the stressful events and the onset of Cushing's syndrome. These facts explain the multifactorial model of the pathogenesis of Cushing's syndrome as a result of the complex interplay between neurophysiological, biochemical and behavioral factors [17].

The delay of the diagnosis of Cushing's syndrome hides the risk not only for another suicide attempt but also for somatic complications with an unfavorable outcome in this particular case - sepsis. The suicidal risk stayed high even after the surgical treatment and it only decreased after the Hydrocortisone was stopped and the serum cortisol levels had normalized. 
The psychic symptoms' progression demonstrates a dynamic, consisting of a severe depressive episode with atypical clinical signs, an expression of unpredictable and psychotically motivated disorganized behavior up until the operative treatment. Predominant psychotic symptoms are: a mask-like facial expression, facies oleosa, active negativism, impulsivity, paralogical thought process, a lack of insight. Part of the described symptoms are similar to those in the catatonic syndrome. The psychotic symptoms display a dynamic within the 24 -hour period. The most severe episodes of sudden psychomotorical agitation, accompanied by self-harm attempts (auto-aggression), occur mainly in morning hours. These conditions resemble a raptus, manifested with impulsive and unconsidered actions, during which the patient remains silent - the so-called "mute agitation" [27]. After the surgical treatment, the symptoms of atypical depression remain predominant.

The normalization of the cortisol levels was the major cause for the successful treatment of the depression in Cushing's syndrome. It was assumed that the rising cortisol levels, that worsen the patient's mental state, were the cause for the lack of efficiency of the antidepressant treatment. The initially applied Venlafaxine in doses of $225 \mathrm{mg} /$ day resolved the symptoms of depression to an insignificant extent and did not induce the manifestation of manic and hypomanic symptoms. Until the normalization of the serum cortisol levels the suicidal thoughts and plans persisted. Studies have shown that an improvement of the depressive symptoms in Cushing's syndrome can be achieved by administering drugs, which reduce synthesis or suppress the effects of cortisol such as Metyrapone, ketoconazole or Mifepristone [28,15]. Therefore, registering atypical signs of depression, accompanied by persistent suicidal thoughts, intentions and plans, and the presence of therapeutic resistance to antidepressant therapy, may guide clinicians to their exogenous origin in search of somatic signs.

The increase of serum cortisol levels leads to significant dynamics in the mental state - the onset of psychotic symptoms resistant to antipsychotic treatment. This observation corresponds to the described cases of cortisol-induced psychoses, in which the psychotic production is not affected by the antipsychotic treatment [29]. Partial sedation is reported with Antiallerzine up to $75 \mathrm{mg} /$ day. Timely surgical treatment is crucial, considering the rapid rise in cortisol levels, the missing rhythm of plasma cortisol and subtle ACTH. These objective indicators could explain the sudden change in behavior with leading psychotic production.

After the removal of the adenoma, treatment with Aripiprazole $15 \mathrm{mg} /$ day is initiated, given the presence of amenorrhea and the centripetal distribution of adipose tissue. Persistent depressive symptoms require re-administration of an antidepressant - in this case, Duloxetine $90 \mathrm{mg} /$ day - an inhibitor of CYP2D6, which decreases the dose of Aripiprazole to $5 \mathrm{mg} /$ day $[30,31]$. The hypertension was treated with Verapamil hydrochloride SR $240 \mathrm{mg} /$ day and substitution therapy with Hydrocortisone was applied.

Is it necessary to continue the psychiatry treatment after normalization of cortisol levels and finding out the somatogenic origin of depression? Research by D. Dorn et al found that after correction of hypercortisolism, atypical depression remained the predominant psychiatric disorder, and the incidence of suicidal ideation even increased [12]. These data can be explained by structural and functional changes in the brain due to prolonged hypercortisolemia [16]. Another factor that requires continuous supporting treatment and systemic monitoring of the patient's condition is the presence of a genetic predisposition i.e. an additional vulnerability of the brain parenchyma to the manifestation of mental disorders. It is important to stimulate protective factors - the supportive social environment and resilience as a personal characteristic against stressors. In this regard, the psychotherapeutic work with both the patient and their family is crucial.

Cortisol's role as a response to various stress factors in the pathophysiology of the endogenic psychoses is unclear and not fully confirmed. Scientists, however, have found that parameters, such as anxiety and stress intolerance index are positively correlated to cortisol levels [32]. In the described case, the regular monitoring of the condition is extremely important for the detection of possible prodromes of psychiatric disorder, in which endogenous pathogenetic mechanisms are superimposed.

In the past, some authors (Bonhöffer) have supported the concept of the total opposition of exogenic and endogenic psychoses, thus creating a "chasm" between them. Others, however, (Snezhnevsky, Goldberg, Malkin, etc.) have supported the thesis of the unity of exogenous and endogenous psychoses, which necessitated the need for an identical approach in both groups of psychiatric disorders [27].

\section{CONCLUSION:}

Cushing's syndrome is a rare and difficult to diagnose and treat. Along with the typical somatic symptoms, the disease is associated with a variety of different psychopathological symptoms. Depression, often presented with an atypical clinical manifestation, is among the most common psychiatric disorders, therapeutically resistant to antidepressant treatment. Depressive episodes can be one of the first manifestations of the disease, can occur and be a significant symptom of the full clinical presentation of the disease or even can be seen during the remission period. In the cases, in which the depression develops during the prodromal period and the somatic symptoms are less apparent, there is a risk of misdiagnosis, delayed diagnosis and life-threatening (incl. autoaggressive) complications. This requires a systemic assessment of the depressive symptoms' severity and the suicide risk during all stages of the disease. The severity of the symptoms corresponds to the severity of the hormonal imbalance. The effective collaboration between endocrinologists, surgeons and psychiatrists in the processes of diagnosing and treatment in all stages of the disease is crucial. Psychotherapy during remission, which aims to stimulate protective factors, along with the biological treatment of the depression, is of key importance to reducing the suicidal risk and improving the quality of life of patients with Cushing's syndrome. 


\section{REFERENCES:}

1. Sievers C, Stalla GK. Psychiatric Disease in Hypothalamic-Pituitary Disorders. In: The Pituitary. 4th Edition. Academic press. 2017. Section II. Chapter 14. pp.413-419. [Crossref]

2. Arnaudova-Zhekova MD. Pathophysiological, clinical diagnostic and therapeutic aspects of delirium. University Publishing House of Medical University - Varna. 2017; 26-44.

3. Steinberg H, Kirkby KC, Himmerich. The Historical Development of Immunoendocrine Concepts of Psychiatric Disorders and Their Therapy. Int J Mol Sci. 2015 Dec 4; 16(12):28841-69. [PubMed]

4. Laane CL. Cushing's syndrome associated with obliterative arterial disease and multiple subcutaneous nodules. (Ehlers-Danlos syndrome?) Acta Med Scand. 1954;148(4):323-5. [PubMed]

5. Newell-Price JDC. Cushing Disease. In: The Pituitary. 4th Edition. Academic Press. 2017. Section III. Chapter 17. pp.515-571. [Crossref]

6. Hirsch D, Shimon I, Manisterski Y, Aviran-Barak N, Amitai O, Nadler V, et al. Cushing's syndrome: comparison between Cushing's disease and adrenal Cushing's. Endocrine. 2018 Dec; 62(3):712-720. [PubMed]

7. Nieman LK. Recent Updates on the Diagnosis and Management of Cushing's Syndrome. Endocrinol Metab (Seoul). 2018 Jun;33(2):139146. [PubMed]

8. Alwani RA, Schmit Jongbloed LW, de Jong FH, van der Lely AJ, de Herder WW, Feelders RA. Differentiating between Cushing's disease and pseudo-Cushing's syndrome: comparison of four tests. Eur J Endocrinol. 2014 Mar 8;170(4):477-86. [PubMed]

9. Findling J, Raff H. Diagnosis of endocrine disease: Differentiation of pathologic/neoplastic hypercortisolism (Cushing's syndrome) from physiologic /non-neoplastic hypercortisolism (formerly known as pseudo-Cushing's syndrome). Eur $J$ Endocrinol. 2017 May;176(5):R205R216. [PubMed]

10. Starkman MN, Schteingart DE, Schork MA. Depressed mood and other psychiatric manifestations of Cushing' syndrome: relationship to hormone levels. Psychosom Med. 1981 Feb;43(1):3-18. [PubMed]

11. Dorn LD, Burgess ES, Dubbert B, Simpson SE, Friedman TC, Kling $\mathrm{M}$, et al. Psychopathology in patients with endogenous Cushing's syndrome: 'atypical' or melancholic features. Clin Endocrinol (Oxf). 1995 Oct;43: 433-42. [PubMed]

12. Dorn LD, Burgess ES, Friedman TC, Dubbert B, Gold PW, Chrousos GP. The longitudinal course of psychopathology in Cushing's syndrome after correction of hypercortisolism. J Clin Endocrinol Metab. 1997; 82(3): 912-9. [PubMed]

13. Haskett RF. Diagnostic categorization of psychiatric disturbance in Cushing's Syndrome. Am J Psychiatry. 1985 Aug;142(8):911-6. [PubMed]

14. Raff H. Cushing Syndrome: Update on Testing. Endocrinol Metab Clin North Am. 2015 Mar;44(1):4350. [PubMed]

15. Arnaldi G, Angeli A, Atkinson AB, Bertagna $\mathrm{X}$, Cavagnini F, Chrousos GP, et al. Diagnosis and complications of Cushing's syndrome: a consensus statement. J Clin Endocrinol Metab. 2003 Dec;88(12): 5593-602. [PubMed]

16. Pivonello R, Simeoli C, De Martino MC, Cozzolino A, De Leo M, Iacuaniello D, et al. Neuropsychiatric disorders in Cushing's syndrome. Front Neurosci. 2015 Apr 20;9:129. [PubMed]

17. Sonino N, Fava GA, Raffi AR, Boscaro M, Fallo F. Clinical correlates of major depression in Cushing's disease. Psychopathology. 1998 NovDec;31(6):302-6. [PubMed]

18. Loosen PT, Chambliss B, DeBold CR, Shelton R, Orth DN. Psychiatric phenomenology in Cushing's disease. Pharmacopsychiatry. 1992 Jul;25(4):192-198. [PubMed]

19. Lojko D, Rybakowski JK. Atypical depression: current perspectives. Neuropsychiatr Dis Treat. 2017 Sep 20;13:2447-2456. [PubMed]

20. Ivanov VB. [Differential diagnosis of psychiatric disorders.] [in Bulgarian] Sofiia: Meditsina i fizkultura. 1991. pp.108-198.

21. Tsai W-T, Tsai S-J, Yang AC. Cyclic Cushing's syndrome mimicking bipolar disorder. Psychiatry and Clinical Neurosciences. 2016 Jan; 70(1):71. [PubMed]

22. Claser GH. Psychotic reactions induced by corticotropin (ACTH) and cortisone. Psychosom Med. 1953 JulAug; 15(4):280-91. [PubMed]

23. Kelly WF. Psychiatric aspects of Cushing's syndrome. QJM. 1996 Jul;89(7):543-51. [PubMed]

24. Hirsch D, Orr G, Kantarovich V, Hermesh H, Stern E, Blum I. Cushing's syndrome presenting as a schizophrenia-like psychotic state. Isr J Psychiatry Relat Sci. 2000; 37(1):4650. [PubMed]

25. Dong TS, Henry JT, Stanley K, Pannain S. Catatonia Induced by an ACTH-Secreting Neuroendocrine Tumor: A Case Report. AACE Clinical Case Reports. 2015 Autumn; 1(4):e245-e249. [Crossref]

26. Brzana J, Yedinak CG, Hameed N, Plesiu A, McCartney S, Fleseriu M. Polycystic ovarian syndrome and Cushing's syndrome: a persistent diagnostic quandary. Eur J Obstet Gynecol Reprod Biol. 2014 Apr;175: 145-8. [PubMed]

27. Zaimov K. [Psychopathology. Lecture course for psychology students.] [in Bulgarian] Science and art, Sofia. 1971; 103-126.

28. Pereira AM, Tiemensma J, Romijn JA. Neuropsychiatric Disorders in Cushing's Syndrome. Neuroendocrinology. 2010; 92(suppl 1):6570. [PubMed]

29. Bilgin YM, van der Wiel HE, Fischer HR, De Herder WW. Treatment of severe psychosis due to ectopic Cushing's syndrome. J Endocrinol Invest. 2007 Oct;30(9): 776-9. [PubMed]

30. Knadler MP, Lobo E, Chappell J, Bergstrom R. Duloxetine: clinical pharmacokinetics and drug interactions. Clin Pharmacokinet. 2011 May;50(5):281-94. [PubMed]

31. Zhang X, Xiang Q, Zhao X, Ma L, Cui Y. Association between aripiprazole pharmacokinetics and CYP2D6 phenotypes: A systematic re- 
view and meta-analysis. J Clin Pharm Ther. 2019 Apr;44(2):163-173. [PubMed]

32. Karanikas E, Garyfallos G. Role of cortisol in patients at risk for psychosis mental state and psychopathological correlates: A systematic review. Psychiatry Clin Neurosci. 2015 May;69(5):268-82. [PubMed]

Please cite this article as: Telbizova T, Aleksandrov I, Arnaoudova M. Dynamics of psychiatric symptoms in Cushing's syndrome. J of IMAB. 2020 Oct-Dec;26(4):3443-3448. DOI: https://doi.org/10.5272/jimab.2020264.3443

Received: 17/11/2019; Published online: 19/11/2020

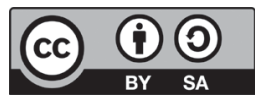

Address for correspondence:

Tatyana Telbizova MD

Department of psychiatry and medical psychology, MHAT St. Marina, Medical University-Varna,

1, Hristo Smirnenski Str., 9010 Varna, Bulgaria.

E-mail: ttelbizova@gmail.com 